\title{
DIMENSIONES DE PERSONALIDAD Y POTENCIAL EVOCADO CEREBRAL
}

\author{
S. CAMPOSANO, C. ALVAREZ, F. LOLAS
}

\begin{abstract}
RESUMEN - La teoría de la personalidad de Eysenck postula 3 dimensiones ortogonales de personalidad: extraversión (E), neuroticismo (N), psicoticismo (P). Formula predicciones conductuales y fisiológicas relacionándolas a la predisposición a ciertos trastornos. La base biológica de las dimensiones $\mathrm{E}$ y $\mathrm{N}$ se ha evidenciado en diferencias electrofisiológicas. La dimensión P, agregada posteriormente, ha sido más controvertida, postulándose que no es independiente de las dimensiones antes descritas. Con objeto de estudiar las dimensiones de Eysenck, en particular P, en relación a la reactividad sensorial y a procesos de codificación se registró potencial evocado visual (PEV) por flash a 3 diferentes intensidades y P300 auditiva en 20 voluntarios sanos ( $\bar{x} 28,5$; ds 9,4 años). Se midió amplitud peak to peak y latencia de los componentes III, IV-V-VI y VII de PEV y de P300 mediante programa cursor. Hubo correlación positiva entre dimensiones $\mathbf{N}$ y $\mathbf{P}$ (spearman, $r=0,52)$, entre $P$ y amplitud del PEV $(r=0,58)$ a intensidad alta y negativa entre E y latencia de $P 300(r=-0,58)$. Según nuestros hallazgos, la dimensión $P$ no es independiente y demostró relación con la reactividad sensorial. La dimensión $\mathrm{E}$ se relacionó a la velocidad de codificación, apoyando las aseveraciones de Eysenck sobre memoria y aprendizaje.
\end{abstract}

PALABRAS CLAVE: personalidad, dimension, potencial evocado cerebral.

\section{Personality dimensions: electrophysiological correlates}

SUMMARY - Eysenck's personality theory postulates 3 orthogonal dimensions of personality: extraversion $(E)$, neuroticism (N) and psychoticism (P), predicting conductual and physiological predispositions to suffer mental illness. Biological bases of Eysenck's personality traits have been documented electrophysiologically. Psychoticism, the latest described dimension, is controverted, since there is some evidence of common factors with the other two. In order to assess the relation between Eysenck's dimensions and sensorial reactivity and information encoding processes we studied 20 healthy young subjects $(\bar{x}$ age 28.5 years) with flash visual cortical evoked potentials (VEP, 3 intensities, peak to peak amplitude of III, IV-V-VI, VII components), and auditory cognitive evoked potentials (odd ball paradigm, P300 latency). There was a positive correlation between $\mathrm{N}$ and $\mathrm{P}$ dimensions (Spearman, $\mathrm{r}=0.52$ ), between $\mathrm{N}$ and VEP amplitude at high intensity $(r=0.58)$ and a negative correlation between $E$ and $\mathrm{P} 300$ latency ( $r=-0.58)$. In short we found that $\mathrm{P}$ is not an independent dimension, but is related to sensorial reactivity. E dimension was related to encoding processes supporting Eysenck's aseverations about memory and learning differences.

KEY WORDS: personality, dimension, evoked brain potencials.

Históricamente la teoría de la personalidad ha sido nutrida por diferentes corrientes. En lo biológico la teoría de Eysenck se ha relacionado con múltiples indicadores conductuales y fisológicos ${ }^{3}$. Numerosos estudios han buscado relacionar las dimensiones de extroversión (E), neuroticismo (N)

Unidad de Psicofisiología, Departamento de Fisiología y Biofísica, Faculdad de Medicina, Universidad de Chile. Financiado mediante proyectos FONDECYT 1090 - 92 y U. de Chile B 3203-9222. Aceite: 16-abril-1994.

Dr. Fernando Lolas - Unidad de Psicofisiología, Depto. de Fisiologia y Biofísica, Facultad de Medicina, Universidad de Chile - Casilla 70055 - Santiago 7 - Chile. 
y psicoticismo (P) con la función de diversas estructuras o neurotransmisores del SNC. Por ejemplo Lester ${ }^{7}$ relacionó niveles de dopamina con la dimensión psicoticismo, niveles de serotonina con neuroticismo y norepinefrina con extraversión (antiguamente englobado en el concepto de función reticular activante). Estas relaciones no son tan simples, existe interacción entre ambos sistemas, cuyo balance determinaría la respuesta conductual y son modificados por neuropéptidos que actúan como neuromoduladores ${ }^{9}$. En cuanto a correlatos electrofisiológicos se ha descrito diferencias en la reactividad electrocortical como amplitud de potenciales evocados y en pendientes de aumentoreducción del potencial evocado visual (PEV) y potencial evocado auditivo (PEA). Estos estudios incluyen correlaciones entre PE y diferentes escalas de "búsqueda de sensaciones"2, "aumentoreducción conductual" "y dimensiones de personalidad". Diferencias en aumento-reducción de los potenciales evocados también se han descrito en animales constituyendo un interesante modelo que parece apoyar la hipótesis noradrenérgica (locus coeruleus) como mediador de la sincronización de respuestas corticales difusas implicadas en este fenómeno ${ }^{10}$. La dimensión más controvertida, ya que fue descrita más recientemente, es psicoticismo. Se ha puesto en duda su independencia de las otras dos y no hay acuerdo en cuanto al correlato electrofiosiológico ${ }^{4}$.

El objetivo de este trabajo es aportar datos al estudio de la base biológica de las dimensiones de Eysenck, especialmente la dimensión $\mathrm{P}$, mediante su relación con potenciales visuales corticales evocados por flash. Nos proponemos además estudiar la posible relación con potenciales evocados cognitivos (P300 auditiva), que se han relacionado especialemnte con funciones cognitivas y con enfermedades psiquiátricas: esquizofrenia y depresión endógena, pero no directamente con dimensiones de Eysenck ${ }^{15}$.

\section{MATERIALES Y METODO}

Sujetos - 20 voluntarios sanos, 6 mujeres y 14 hombres, edad promedio 28,5 años (d.s.9,4 años), sin antecedentes de patología neurológica o psiquiátrica.

Protocolo experimental - todos los sujetos fueron sometidos a:

1. Prueba de Eysenck (EPQ-R), en su versión española de 101 preguntas.

2. Potencial evocado visual por flash, intervalo interestímulo 1 seg y 3 intensidades $0,18,0,36$ y 0,72 Joules a $1 \mathrm{~m}$ de distancia con ojos cerrados. Se registró EEG en derivaciones $\mathrm{Oz}-\mathrm{Cz}$ y electrooculograma (EOG) (supraciliar y canto) con amplificación de 10000 veces y filtro pasabanda 1-100 Hz. Se promediaron 100 respuestas para cada intensidad, con tiempo de registro de $300 \mathrm{mseg}$. Se midió amplitud peak to peak y latencia de los componentes III, IV-V-VI y VII

3. Potencial evocado auditivo P300 en paradigma Odd-Ball en tarea de cómputo mental para tres paradigmas de diferente dificultad. Se instruyó a los sujetos a contar mentalmente los tonos agudos. Las tres series fueron de 300 estímulos con un $20 \%$ de estímulos "target" (ET) y un $80 \%$ de estímulos "standard" (ES), ambos binaurales, con intensidad de $80 \mathrm{~dB}$ SPL y duración de $30 \mathrm{mseg}$. La Tarea 1 (mayor dificultad) constó de: ET de $1500 \mathrm{~Hz}$ y ES de $1400 \mathrm{~Hz}$, intervalo interestímulo de $1 \mathrm{seg}$. Tarea 2 (dificultad intermedia): ET $1500 \mathrm{~Hz}$, ES $750 \mathrm{~Hz}$, intervalo interestímulo $1 \mathrm{seg}$. Tarea 3 (fácil): ET $1500 \mathrm{~Hz}$, ES $750 \mathrm{~Hz}$, intervalo interestímulo $1,5 \mathrm{seg}$.

Se registró EEG en derivaciones $\mathrm{Cz}, \mathrm{Pz}$ y $\mathrm{Fz}$ referidas a mastoides y EOG supraciliar y canto, con un tiempo de registro de $600 \mathrm{mseg}$. Se usaron filtros de 1 a $30 \mathrm{~Hz}$ y amplificación de 20000 veces. Se promedió separadamente la respuesta al ET (60) y al ES(240) para cada tarea. Se eliminaron del análisis todos aquellos registros en que la amplitud "peak to peak" del EOG

Tabla 1. Coeficientes de correlación segun Spearman.

\begin{tabular}{lccc}
\hline & $\mathrm{E}$ & $\mathrm{N}$ & $\mathrm{P}$ \\
\hline \hline $\mathrm{P}$ & -0.12 & $0.52^{*}$ & - \\
Ampl III-IV int 0,72 J & 0.21 & 0.38 & $0.57^{*}$ \\
Lat P300 tarea 1 & $-0.57^{*}$ & -0.41 & -0.19 \\
\hline
\end{tabular}
fue mayor que en $\mathrm{Cz}$ (artefacto ocular). Se midió latencia de $\mathrm{P} 300$ mediante programa cursor.

\section{RESULTADOS}

Se investigó correlaciones no paramétricas mediante el método de Spearman (Tabla 1). Hubo correlación significativa entre las dimensiones $\mathbf{N}$ y $\mathrm{P}$ $(0,52)$, entre la amplitud del componente III-IV a 0,72 Joules y $\mathrm{P}(0,57)$ (Fig 1) y correlación negativa entre latencia de $\mathrm{P} 300$ en tarea 1 (de difícil discriminación entre estímulos) y dimensión E (Fig 3). 
Tabla 2. Amplitudes "peak to peak" (uV) del potencial evocado visual, componente III-IV; Wilcoxon $* p<0.03$.

\begin{tabular}{|c|c|c|}
\hline Segun E & $\mathrm{E}$ alto $(>=12)$ & E bajo $(<12)$ \\
\hline $\begin{array}{l}\text { int } 0.18 \mathrm{~J} \\
\text { prom } \\
\text { ds }\end{array}$ & $\begin{array}{c}14.3 \\
8.9\end{array}$ & $\begin{array}{c}16.2 \\
5.2\end{array}$ \\
\hline $\begin{array}{l}\text { int } 0.36 \mathrm{~J} \\
\text { prom } \\
\text { ds }\end{array}$ & $\begin{array}{l}14.7 \\
10.5\end{array}$ & $\begin{array}{c}17.0 \\
5.2\end{array}$ \\
\hline $\begin{array}{l}\text { int } 0.72 \mathrm{~J} \\
\text { prom } \\
\text { ds }\end{array}$ & $\begin{array}{l}18.7 \\
10.4\end{array}$ & $\begin{array}{c}16.2 \\
5.4\end{array}$ \\
\hline Segun $P$ & $\mathrm{P}$ alto $(>=7)$ & P bajo $(<7)$ \\
\hline $\begin{array}{l}\text { int } 0.18 \mathrm{~J} \\
\text { prom } \\
\text { ds }\end{array}$ & $\begin{array}{c}17.2 \\
8.1\end{array}$ & $\begin{array}{c}13.9 \\
5.6\end{array}$ \\
\hline $\begin{array}{l}\text { int } 0.36 \mathrm{~J} \\
\text { prom } \\
\text { ds }\end{array}$ & $\begin{array}{c}18.4 \\
8.4\end{array}$ & $\begin{array}{c}13.5 \\
6.7\end{array}$ \\
\hline $\begin{array}{l}\text { int } 0.72 \mathrm{~J} \\
\text { prom } \\
\text { ds }\end{array}$ & $\begin{array}{c}20.9 \\
9.6\end{array}$ & $\begin{array}{c}14.5 \\
5.3\end{array}$ \\
\hline
\end{tabular}

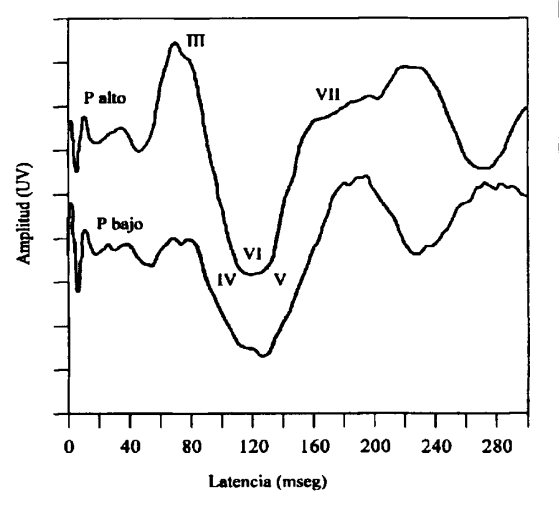

Fig 1. Gran promedio P.E.V. int 8.
Tabla 3. Amplitudes "peak to peak" (uV) del potencial evocado visual, componente IV-VII; Wilcoxon ${ }^{* *} p<0.03$.

Segun E E alto $(>=12) \quad$ E bajo $(<12)$ int $0.18 \mathrm{~J}$

$\begin{array}{lcc}\text { prom } & 16.4 & 19.7 \\ \text { ds } & 7.2 & 5.1\end{array}$

int $0.36 \mathrm{~J}$

$\begin{array}{lcc}\text { prom } & 13.9 * * & 21.1 \\ \text { ds } & 6.9 & 5.5\end{array}$

int $0.72 \mathrm{~J}$

\begin{tabular}{lcc} 
prom & 17.8 & 19.2 \\
ds & 7.1 & 6.3 \\
\hline
\end{tabular}

Tabla 4. Promedio de latencia de P300 (ms).

E alto $(>=12) \quad$ E bajo $(<12)$

Tarea 1

$\begin{array}{lcc}\text { prom } & 292 & 318 \\ \text { ds } & 20 & 24\end{array}$

Tarea 2

prom $265 \quad 273$

$\begin{array}{lll}\text { ds } & 38 & 29\end{array}$

Con objeto de demostrar gráficamente las diferencias en los PE existentes entre sujetos con puntajes altos y bajos para cada dimensión, se dividió la muestra en 2 grupos con igual número de sujetos sgún sus puntajes en cada dimensión (Tabla 2). Estas diferencias se estudiaron estadísticamente mediante el método de Wilcoxon (Tablas 3 y 4). Hubo diferencias significativas en la amplitud peak to peak del componente VI-VII del PE visual a 0,36 Joules para los sujetos con E alto y E bajo (Fig 2).

\section{COMENTARIOS}

La dimensión $\mathbf{P}$ demostró relación con la amplitud de los potenciales evocados visuales: hubo correlación significativa entre la amplitud 


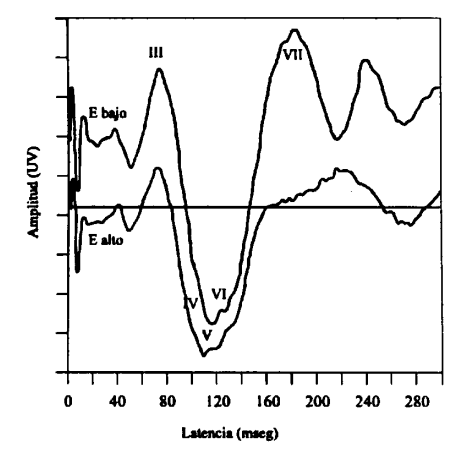

Fig 2. Gran promedio P.E.V. int 4.

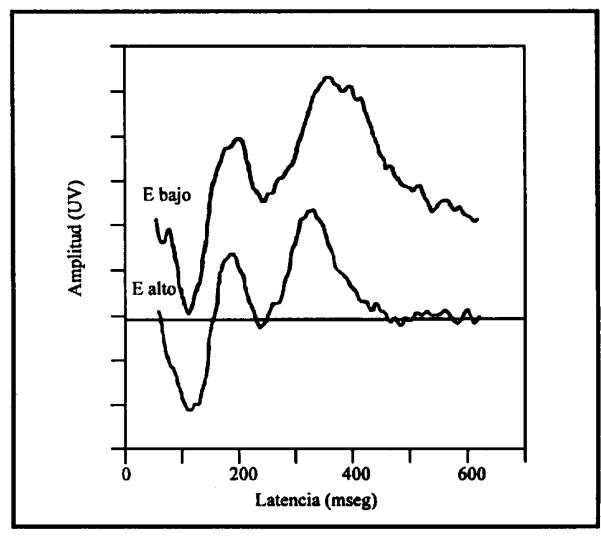

Fig 3. P.E. cognitivos, E alto, E bajo.

del componenete III-IV del PEV a intensidad máxima y dimensión P. Esta relación es coherente con las teorías neuroquímicas, ya que la vía visual presenta un importante componente dopaminérgico y el complejo III-IV refleja la activación cortical temprana. En cuanto a la independencia de la dimensión $\mathrm{P}$, ésta no pudo ser confirmada: hubo correlación significativa entre $\mathrm{N}$ y $\mathrm{P}$; planteando la necesidad de una revisión de los rasgos que se asignan a $P$.

Se confirma la mayor reactividad sensorial de los sujetos introvertidos ${ }^{11}$ : mayor amplitud del componente VI-VII en el grupo con bajo $\mathrm{E}$ a intensidad media. Este componente es también de origen cortical y siendo más tardío refleja mayor procesamiento.

Los potenciales evocados cognitivos demostraron relación con la dimensión E. Cabe destacar que sólo hubo correlación para la tarea más dificil (tarea 1), que significa una mayor demanda atencional, ya que se trata de identificar estímulos de tonalidad parecida $(1400$ y $1500 \mathrm{~Hz}) \mathrm{con}$ intervalo interestímulo corto ( $1 \mathrm{seg}$ ). Los extrovertidos presentan latencia menor que los introvertidos. Este hallazgo refleja diferencias en el proceso atencional" en el sentido de una mayor velocidad en la categorización de la información ${ }^{6}$ por los sujetos extrovertidos.

En suma podemos afirmar que existen evidencias electrofisiológicas de una base biológica para $\mathrm{P}$ aunque ésta no haya demostrado independencia de $\mathrm{N}$. Los potenciales cognitivos (P300) están influenciados por factores de personalidad, dada su relación con los puntajes de $\mathrm{E}$.

\section{REFERENCIAS}

1. Bruder G. P300 finding for depressive and anxiety disorders. Ann NY Acad Sci. 1992, Vol 658.

2. Carrillo-de-la-Peña MT. REP augmenting-reducing sensations seeking: a critical review. Int J Psychophysiol 1992, 12: 212-220.

3. Etcheberrigaray R, Lolas F, Camposano S, Elgueta D. Topografía y reactividad del potencial evocado visual. Arq Neuropsiquiatr 1988, 46: 258-263.

4. Eysenck HJ. A model for personality. Berlin: Springer Verlag, 1981.

5. Ford J, Pfefferbaum A, Roth W. P3 and schizophrenia. Ann NY Acad Sci 1992, Vol 658.

6. Kutas M, McCarthy G, Donchin E. Augmenting mental chronometry: the P300 as a measure of stimulus evaluation time. Science 1977, 197: 792-795.

7. Lester D. A neurotransmitter basis for Eysenck's theory of personality. Psychol Report 1989, 64: 189-190.

8. Lolas F, Camposano S, Etcheberrigaray R. Augmenting/reducing and personality: a psychometric and evoked potential study in a chilean sample. Personal Indiv Diff 1989, 11: 1173-1176.

9. Mulder R. The biology of personality. Aust N Z J Psychiatry 1992, 26: 364-376.

10. Pineda JA, Holmes TC, Foote SL. Intensity-amplitude relationships in monkey event related potencials: parallels to human augmenting-reducing responses. Electroenceph Clin Neuophysiol 1991, 78: 456-465.

11. Stelmack RM. Advances in personality theory and research. J Psychiat Neurosci 1991, 16: 131-138. 